\title{
Identification of Broadband Source-Array Responses from Sensor Second Order Statistics
}

\author{
S. Weiss*, N.J. Goddard ${ }^{\dagger}$, S. Somasundaram ${ }^{\ddagger}$, I.K. Proudler ${ }^{*}$, , and P.A. Naylor \\ *Dept. of Electronic \& Electrical Engineering, University of Strathclyde, Glasgow G1 1XW, Scotland \\ ${ }^{\dagger}$ DSTL Portsdown, Fareham, Hampshire PO17 6AD, UK \\ ¥Thales Underwater Systems, Cheadle, Stockport SK3 0XB, UK \\ $\S$ School for Mechanical, Electrical and Manufacturing Eng., Loughborough University, Leicestershire LE11 3TU, England \\ IDept. of Electrical \& Electronic Engineering, Imperial College London, London SW7 2AZ, UK \\ e-mail: stephan.weiss@ strath.ac.uk
}

\begin{abstract}
This paper addresses the identification of sourcesensor impulse responses from the measured space-time covariance matrix in the absence of any further side information about the source or the propagation environment. Using polynomial matrix decomposition techniques, the responses can be narrowed down to an indeterminacy of a common polynomial factor. If at least two different measurements for a source with constant power spectral density are available, this indeterminacy can be reduced to an ambiguity in the phase response of the sourcesensor paths.
\end{abstract}

\section{INTRODUCTION}

Second order statistics of sensor array data have been used in numerous ways to characterise signal processing parameters. In the case of sources in the array's far-field, and in the absence of multipath propagation, for example the angles of arrival can be estimated for both narrowband [1], [2] and broadband signals [3]-[5]. For near-field or multipath propagation, signal parameters have been extracted for the narrowband case, see e.g. [6], but the broadband approach is more difficult and often can only be made sufficiently robust to multipath effects without directly exploiting or extracting these [7].

However, the extraction of parameters such as a source's power spectral density (PSD), as well as multipath characteristics of the transfer paths can be usefully exploited to obtain clues about the propagation environment, which in turn can assist in locating a source. For example, in [8] a model of the propagation environment has been extracted from a single impulse response. Similarly, impulse responses of a single input multiple output sysyte can be utilised to infer the geometry of an acoustic room [9] or attempt to acoustically image the propagation environment and locate the source [10].

Therefore, this paper investigates to which extend polynomial matrix decomposition techniques [11] can assist in resolving the desired impulse responses. To do this, Sec. II describes the model for the scenario, and the data that is acquired. Sec. III reviews the polynomial eigenvalue decomposition (PEVD) and ambiguities associated with its polynomial matrix factors. Sec. IV outlines that with the source in a single position, not much can be determined. If measurements include a relocation of the source (by either movement of the source or the sensor array), then the source power spectral density and the magnitude responses of the transfer paths can be extracted, as demonstrated in Sec. V. While this leaves us with a phase ambiguity, the polynomial approach is still capable to retrieve significantly more information than a frequency-bin approach would be capable of achieving, as shown in Sec. VI. A numerical example is provided in Sec. VII.

\section{SOURCE MOdEL}

We assume a single source that illuminates an $M$-element sensor array as shown in Fig. 1. The transfer functions between the source and the sensor elements are contained in a vector $\boldsymbol{a}_{i}(z) \in \mathbb{C}^{M}$,

$$
\boldsymbol{a}_{i}(z)=\left[\begin{array}{c}
A_{i, 1}(z) \\
\vdots \\
A_{i, M}(z)
\end{array}\right],
$$

where $i$ is a measurement campaign index. A measurement campaign is here defined as a data acquisition over a brief period of time over which the propagation paths between source and array are stationary, such that transfer functions as shown in (1) can be defined. The source is assumed to be wide sense stationary with a PSD $S(z)$. From one measurement campaign to the next, a variation of the transfer functions in $\boldsymbol{a}_{i}(z)$ is expected to occur and may arise from a relative change between the source and the sensor array, i.e. either through a movement of the source, a change in the array's position, orientation, or configuration, or through a change in the propagation environment.

If the sensor signals are collected in a vector $\mathbf{x}_{i}[n] \in$ $\mathbb{C}^{M}$, then the sensor covariance matrix is $\mathbf{R}_{i}[\tau]=$ $\mathcal{E}\left\{\mathbf{x}_{i}[n] \mathbf{x}_{i}^{\mathrm{H}}[n-\tau]\right\}$. The cross-spectral density (CSD) during this $i$ th campaign is

$$
\begin{aligned}
\boldsymbol{R}_{i}(z) & =\sum_{\tau=-\infty}^{\infty} \mathbf{R}_{i}[\tau] z^{-\tau} \\
& =\boldsymbol{a}_{i}(z) S(z) \boldsymbol{a}_{i}^{\mathrm{P}}(z)+\sigma_{n}^{2} \mathbf{I}_{M}
\end{aligned}
$$

where $\sigma_{n}^{2}$ is the power of spatially and temporally uncorrelated measurement noise, and $\{\cdot\}^{\mathrm{P}}$ is the so-called parahermitian 


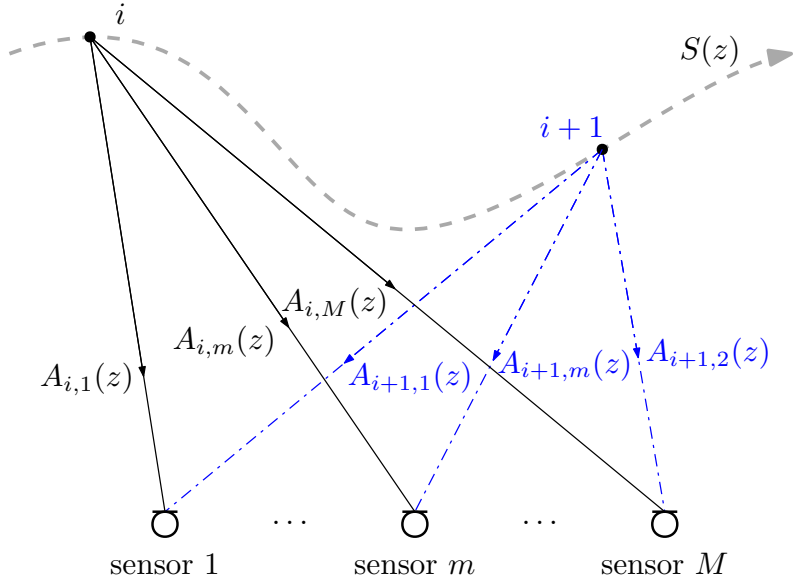

Fig. 1. Model with source power spectral density $S(z)$ and two measurements campaigns along the source's trajectory relative to the sensor array with transfer functions $A_{i, m}(z), m=1 \ldots M$, between the source and the $M$ sensors.

operator, such that $\boldsymbol{a}_{i}^{\mathrm{P}}(z)=\boldsymbol{a}^{\mathrm{H}}\left(1 / z^{*}\right)$ is the Hermitian transposed time-reversed version of $\boldsymbol{a}(z)$. Because of its dependence on $z$, the CSD matrix $\boldsymbol{R}(z)$ is referred to as a polynomial matrix. With $\mathbf{R}[\tau]$ in (3) being composed of autoand cross-correlation terms, due to its inherent symmetry $\boldsymbol{R}(z)$ represents a parahermitian matrix, such that $\boldsymbol{R}(z)=\boldsymbol{R}^{\mathrm{P}}(z)$. This property is an extension of the symmetric or Hermitian property of real and complex-valued matrices, respectively, to the ring of polynomial matrices.

We assume that the source maintains the same power spectral density, but that due to non-stationarity or movement of the source, the vector of transfer functions changes between measurement campaigns.

\section{Polynomial Eigenvalue Decomposition}

The eigenvalue decomposition (EVD) of Hermitian matrices is a central operation in signal processing, and below we will discuss some aspects of defining an extension of the EVD to parahermitian polynomial matrices, called a polynomial EVD (PEVD) [11].

\section{A. Existence}

For a parahermitian matrix $\boldsymbol{R}(z)$, the PEVD currently has not yet been proven to exist, but it is claimed that a good approximation by means of FIR paraunitary matrix factor $\boldsymbol{Q}(z)$ and a diagonal and spectrally majorised $\boldsymbol{\Lambda}(z)$ of sufficient order can be found [12], such that the PEVD [11] or McWhirter decomposition

$$
\boldsymbol{R}(z) \approx \boldsymbol{Q}(z) \boldsymbol{\Lambda}(z) \boldsymbol{Q}^{\mathrm{P}}(z)
$$

holds. Spectral majorisation implies that when evaluating the polynomial eigenvectors contained in $\boldsymbol{\Lambda}(z)=$ $\operatorname{diag}\left\{\lambda_{0}(z) \ldots \lambda_{M-1}(z)\right\}$ on the unit circle, the power spectral densities fulfil

$$
\lambda_{m}\left(e^{j \Omega}\right) \geq \lambda_{m+1}\left(e^{j \Omega}\right) \quad, \forall \Omega \quad m=0 \ldots(M-2) .
$$

This restricts an ambiguity of the decomposition w.r.t. a potential frequency-dependent permutation of eigenvalues and -vectors.

Instead of spectral majorisation, if an analytic $\boldsymbol{R}(z)$ is evaluated on the unit circle, $\boldsymbol{R}\left(e^{j \Omega}\right)=\left.\boldsymbol{R}(z)\right|_{z=e^{j \Omega}}$, Fourier domain factors for eigenvalues and -vectors can be selected analytic in $\Omega$ [13]. These can be extended to eigenvalues and -vectors that are analytic, i.e. maximally smooth, functions in $z$. This now enables an exact decomposition with equality (4), but must be based on Laurent series, since the factors $\Lambda(z)$ and $\boldsymbol{Q}(z)$ may be of infinite length i.e. potentially transcendental functions in $z$. Below, we assume that such an analytic rather than a spectrally majorised PEVD is selected, and exists with equality in (4).

The columns of $\boldsymbol{Q}(z)$ are the polynomial eigenvectors $\boldsymbol{u}_{m}(z)$,

$$
\boldsymbol{Q}(z)=\left[\begin{array}{lll}
\boldsymbol{q}_{0}(z) & \ldots & \boldsymbol{q}_{M-1}(z)
\end{array}\right] .
$$

Based on the polynomial eigenvalues and -vectors, the PEVD can also be written as

$$
\boldsymbol{R}(z) \approx \sum_{m=0}^{M-1} \lambda_{m}(z) \boldsymbol{q}_{m}(z) \boldsymbol{q}_{m}^{\mathrm{P}}(z) .
$$

\section{B. Ambiguity}

To explore ambiguity, we assume that the PEVD in (7) exists with equality. For the eigenvectors,

$$
\boldsymbol{q}_{m}(z) \boldsymbol{q}_{n}^{\mathrm{P}}(z)=\delta(n-m),
$$

holds with $m, n=1 \ldots M$. They can be modified as

$$
\boldsymbol{q}_{m}^{\prime}(z)=U_{m}(z) \boldsymbol{q}_{m}(z),
$$

by an arbitrary allpass filter $U(z)$, such that the $\boldsymbol{q}_{m}^{\prime}(z), m=$ $0 \ldots(M-1)$ will still form valid eigenvectors satisfying (8). This allpass filter must be common to all elements of $\boldsymbol{q}_{m}(z)$, and can in the simplest case form a delay [14]. Note that therefore $\boldsymbol{q}_{m}(z)$ and $\boldsymbol{q}_{m}^{\prime}(z)$ will have an identical magnitude but different phase responses.

This ambiguity is a generalisation of the ambiguity of eigenvectors of a non-polynomial eigenvalue problem, where both sides of $\mathbf{A v}=\lambda \mathbf{v}$ can be multiplied by an arbitrary phase shift $e^{j \varphi}$, such that $\mathbf{v}^{\prime}=\mathbf{v} e^{j \varphi}$ akin to (9).

\section{Iterative PEVD Algorithms}

Iterative PEVD algorithms currently belong to the families of sequential best rotation (SBR2) [11] and sequential matrix diagonalisation (SMD) [15] algorithms. These perform an approximate iterative diagonalisation by FIR paraunitary factors. The type of rational function represented by an allpass filter implementing an arbitrary phase response is likely to be approximated by a large number of zeros. This ambiguity in (8) may be hidden, but motivates the comparison of transfer functions across every eigenvector $\boldsymbol{q}_{m}(z), m=1 \ldots M$ of $\boldsymbol{Q}(z)$ for common zeros, and particular delays [14].

The above PEVD algorithms of the SBR2 and SMD families tend to extract spectrally majorised diagonal matrices, which in 
case of SBR2 can even be proven [16]. In case that eigenvalues intersect on the unit circle, spectral majorisation will enforce the approximation of non-analytic eigenvalues and -vectors. To guarantee analytic eigenvalues even in case of eigenvalues intersecting on the unit circle, algorithms different from SBR2 or SMD are required, with a DFT-base approach in [17] a first step.

\section{Extraction Based on a Single Campaign}

We assume that for a single measurement campaign $i$, the CSD matrix $\boldsymbol{R}_{i}(z)$ according to (2) has been estimated, and its PEVD exists and is known, such that

$$
\begin{aligned}
\boldsymbol{R}_{i}(z) & =\left[\boldsymbol{q}_{i}(z) \boldsymbol{q}_{i}^{\perp}(z)\right]\left[\begin{array}{c}
\gamma_{i}(z)+\sigma_{n}^{2} \\
\sigma_{n}^{2} \mathbf{I}_{M-1}
\end{array}\right]\left[\begin{array}{r}
\boldsymbol{q}_{i}^{\mathrm{P}}(z) \\
\boldsymbol{q}_{i}^{\perp, \mathrm{P}}(z)
\end{array}\right] \\
& =\boldsymbol{q}_{i}(z) \gamma_{i}(z) \boldsymbol{q}_{i}^{\mathrm{P}}(z)+\sigma_{n}^{2} \mathbf{I}_{M} .
\end{aligned}
$$

The difference between the source model in (3) and the PEVD in (11) is that $\boldsymbol{q}_{i}(z)$ is by definition normalised while $\boldsymbol{a}_{i}(z)$ is unnormalised,

$$
\begin{aligned}
\boldsymbol{q}_{i}^{\mathrm{P}}(z) \boldsymbol{q}_{i}(z) & =1 \\
\boldsymbol{a}_{i}^{\mathrm{P}}(z) \boldsymbol{a}_{i}(z) & =A_{i}(z)=A_{i}^{(+)}(z) A_{i}^{(-)}(z) \\
& =A_{i}^{(+)}(z) A_{i}^{(+), \mathrm{P}}(z),
\end{aligned}
$$

where $A_{i}(z)$ is a PSD and $A_{i}[\tau] \circ \longrightarrow A_{i}(z)$ has the symmetry properties of an auto-correlation function, i.e. $A_{i}(z)=A_{i}^{\mathrm{P}}(z)$, and $A_{i}^{(+)}(z)$ is the minimum-phase part of $A_{i}(z)$. It is assumed that $A_{i}(z)$ has no spectral zeros. Normalisation of $\boldsymbol{a}_{i}(z)$ can therefore be accomplished by setting

$$
\boldsymbol{a}_{i, \operatorname{norm}}(z)=\frac{\boldsymbol{a}_{i}(z)}{A_{i}^{(+)}(z)} .
$$

With this normalisation, (3) can be rewritten as

$\boldsymbol{R}_{i}(z)=\boldsymbol{a}_{i, \operatorname{norm}}(z) A_{i}^{(+)}(z) S(z) A_{i}^{(+), \mathrm{P}}(z) \boldsymbol{a}_{i, \text { norm }}^{\mathrm{P}}(z)+\sigma_{n}^{2} \mathbf{I}_{M}$.

Comparing (16) to (11), with $\gamma_{i}(z)$ the dominant eigenvector minus the noise floor in the PEVD in (11), we can extract

$$
\begin{aligned}
\boldsymbol{q}_{i}(z) & =\frac{\boldsymbol{a}_{i}(z)}{A_{i}^{(+)}(z)} \\
\gamma_{i}(z) & =A_{i}^{(+)}(z) S(z) A_{i}^{(+), \mathrm{P}}(z),
\end{aligned}
$$

such that the vector of transfer functions and the source power spectral density can be determined except for unknown scaling factors $1 / A_{i}^{(+)}(z)$ and $A_{i}^{(+)}(z) A_{i}^{(+), \mathrm{P}}(z)$, respectively, and the phase-indeterminacy inherent in the eigenvectors of the PEVD.

\section{Extraction Based on Multiple Campaigns}

Instead of a single campaign, now multiple measurement campaigns have been performed and the decompositions (17) and (18) are available for $i=1 \ldots I$. If there are no roots that are common to all $A_{i}^{(+)}(z)$, then the source PSD represents the greatest common divisor (GCD) across all instances of (18),

$$
\hat{S}(z)=\operatorname{GCD}\left\{\gamma_{1}(z) \ldots \gamma_{I}(z)\right\}
$$

From this, estimates of the scaling terms $\hat{A}_{i}^{(+)}(z)$ remaining in (17) and (18) can be extracted for every measurement campaign.

The estimation of the scaling term $\hat{A}_{i}^{(+)}(z)$ now leads to a more precise estimate of the source-array transfer functions from (17), whereby only an indeterminacy remains in the phase response for every $\hat{\boldsymbol{a}}(z)$ due to the ambiguity characterised in Sec. III-B, leading to

$$
\hat{\mathbf{a}}_{i}(z)=\hat{A}_{i}^{(+)}(z) U_{i}(z) \boldsymbol{q}_{i}(z)
$$

with $U_{i}(z)$ an arbitrary allpass filter according to (9). With the ambiguity restricted to the phase responses, however the magnitude responses of the source-array transfer paths are now fully determined.

The viability of this approach depends on an accurate determination of a GCD, i.e. of finding common zeros across multiple polynomials. This problem has been addressed by Euclid in his 7th book of Elements around 300BC [18], by a method referred to as Euclid's algorithm. Because its robustness deteriorates quickly with the order of the polynomials, refinements are still being made to date, see e.g. [19]-[25].

As we will explore through an example in Sec. VII, extracting a GCD even for a controlled scenario is difficult based on recent algorithms such a [25]. However, the aim here is to highlight the general approach independent of the problem of root finding, where perhaps robust approaches such as Gröbner bases may provide viable future alternatives.

\section{Solution In IndePEndent FREQUenCy Bins}

Classically, broadband problems are often addressed by solving a number of narrowband or discrete Fourier transform (DFT)-domain problems independently in adjacent frequency bins. In this section, we consider an approach where the input signal is split into $K$ such frequency bins, and compare this as a benchmark to the findings of Secs.IV and V.

Applying $K$-point discrete Fourier transforms to the measured signals, then with sufficient averaging $K$ narrowband covariance matrices $\mathbf{R}_{i, k}$ arise at the normalised angular frequencies $\Omega_{k}=\frac{2 \pi k}{K}, k=0 \ldots(K-1)$ during the $i$ th measurement campaign,

$$
\begin{aligned}
\mathbf{R}_{i, k} & =\boldsymbol{R}_{i}\left(e^{j \Omega_{k}}\right)=\boldsymbol{a}_{i}\left(e^{j \Omega_{k}}\right) S\left(e^{j \Omega_{k}}\right) \boldsymbol{a}_{i}^{\mathrm{H}}\left(e^{j \Omega_{k}}\right)+\sigma_{n}^{2} \mathbf{I} \\
& =\mathbf{q}_{i, k} \lambda_{i, k} \mathbf{q}_{i, k}^{\mathrm{H}},
\end{aligned}
$$

where $\boldsymbol{R}_{i}\left(e^{j \Omega_{k}}\right)=\left.\boldsymbol{R}(z)\right|_{z=e^{j \Omega_{k}}}$ is the evaluation of (2) on the unit circle for frequency $\Omega_{k}$.

The principal eigenvectors and eigenvalues for the measurement campaigns are

$$
\begin{aligned}
\mathbf{q}_{i, k} & =\frac{\boldsymbol{a}_{i}\left(e^{j \Omega_{k}}\right)}{\left|\boldsymbol{a}_{i}\left(e^{j \Omega_{k}}\right)\right|}, \\
\lambda_{i, k} & =S\left(e^{j \Omega_{k}}\right)\left|\boldsymbol{a}_{i}\left(e^{j \Omega_{k}}\right)\right|^{2},
\end{aligned}
$$

which again are discrete evaluations of (17) and (18) on the unit circle.

Even for multiple measurement campaigns, the scaling now cannot be resolved, since the coherence between frequency 


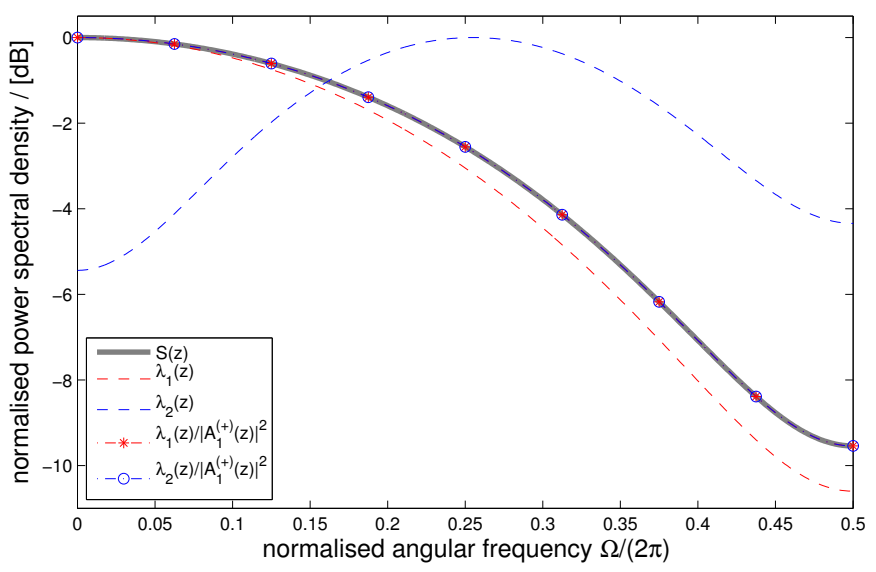

Fig. 2. Source PSD $S(z)$ compared to eigenvalues $\lambda_{i}(z)$ calculated from $\boldsymbol{R}_{i}(z)$ of both measurement campaigns, with the scaled versions to correct for (18). All PSDs are normalised to a maximum value of unity.

bins in the independent frequency bin representation has been lost compared to (17) and (18). Also, (23) has an indeterminacy w.r.t. angle since adding multiples of $2 \pi$ to the phase and therefore imposing a delay will not change the vector. Part of this indeterminacy could be removed by considering coherence across frequency bins and thus unwrapping the phase; this process is however not directly obvious from (23) and (24), and requires additional processing.

Therefore, without additional processing to account for coherence between bins, the independent frequency bin method exhibits an indeterminacy in terms of both amplitude and delay, and can retrieve neither the transfer functions $A_{i, m}(z)$ nor the source power spectral density $S(z)$.

\section{NUMERICAL EXAMPLE}

As an example, a source with power spectral density

$$
S(z)=\frac{1}{2} z+\frac{5}{4}+\frac{1}{2} z^{-1}
$$

illuminates an $M=2$ element array during measurement campaign $i=1$ via the transfer function vector

$$
\boldsymbol{a}_{1}(z)=\left[\begin{array}{ccc}
1 & + & \frac{1}{2} z^{-1} \\
\frac{3}{4} & - & \frac{1}{2} z^{-1}
\end{array}\right],
$$

and during measurement campaign $i=2$ via

$$
\boldsymbol{a}_{2}(z)=\left[\begin{array}{rrr}
\frac{4}{5} & - & \frac{1}{2} z^{-1} \\
-\frac{1}{2} & + & z^{-1}
\end{array}\right]
$$

The PSD and various magnitude responses are shown in Figs. 2-4; for easier comparison with some of the calculations further below, the curves are normalised such that their maximum value is unity.

Using the SMD algorithm [15] to estimate the PEVD, the eigenvalues $\lambda_{i}(z), i=1,2$ are determined; their PSDs are displayed in Fig. 2. These bear no resemblance with the source PSD; neither can the principal eigenvectors $\boldsymbol{q}_{i}(z)$ reveal anything about the source-array transfer functions when considered in isolation. Evaluated on the unit circle, the
TABLE I

RoOTS OF POLYNOMIAL EIGENVALUES $\lambda_{i}(z), i=1,2$.

\begin{tabular}{|r||r|r|}
\hline index & $\lambda_{1}(z)$ & \multicolumn{1}{|c|}{$\lambda_{2}(z)$} \\
\hline \hline 1 & -16.4293 & -1.9998 \\
2 & -2.0001 & 1.8314 \\
3 & -0.5000 & 0.5460 \\
4 & -0.0609 & -0.5001 \\
\hline
\end{tabular}

magnitudes responses of the given source-array paths and the determined principal eigenvectors in Figs. 3 and 4 do not match, as expected from Sec. IV.

To identify the common scaling factors across both campaigns, we closer inspect the eigenvalues $\lambda_{i}(z), i=1,2$. Since the toy problem is noise-free, we have $\gamma_{i}(z)=\lambda_{i}(z)$ w.r.t. (11). After trimming the order of the polynomial matrix factors in the PEVD, the principal eigenvalues campaigns both have four roots each as listed in Tab. I. Two roots at $z=-2$ and $z=-\frac{1}{2}$ match closely, but do not entirely coincide due to estimation errors by the SMD algorithm as well as due to trimming of the polynomial matrix factors [14]. As a results, root finding algorithms such the one in [25] cannot determine the GCD exactly, and roots here have been matched by inspection.

The matching zeros of Tab. I form the GCD, which now represents the estimate for the source PSD according to (19). This is demonstrated by the close agreement between the orginal and the estimated source PSDs in Fig. 2. From the remaining zeros in Tab. I with $|z|<1$, the minimum phase correction factors

$$
\begin{aligned}
& A_{1}^{(+)}(z)=1+0.0609 z^{-1} \\
& A_{2}^{(+)}(z)=1-0.5460 z^{-1}
\end{aligned}
$$

are extracted. These enable the estimation of the transfer function vectors via (20), with ambiguity of the phase response but matching magnitude responses as seen for the paths of campaign $i=1$ in Fig. 3 and for campaign $i=2$ in Fig. 4 .

\section{CONCLUSIONS}

This paper has explored the possibility of extracting sourcesensor paths and source power spectral densities based on the second order statistics of data collected by the sensors only. If only a single measurement is available, the transfer paths have an ambiguity with respect to an unknown common polynomial factor, i.e. neither amplitude nor phase response can be determined precisely. By having at least a second measurement campaign - defined as a separate measurement of the same source but with different transfer functions - this ambiguity can in principle be narrowed down to the phase response. We have assumed that multiple measurement campaigns are taken over time, but this can be equally performed spatially, e.g. by partitioning the array.

The approach has exploited polynomial matrix EVD techniques, which are well-suited for addressing broadband problems, as they address coherence. This has been demonstrated to differ significantly from an independent frequency bin 


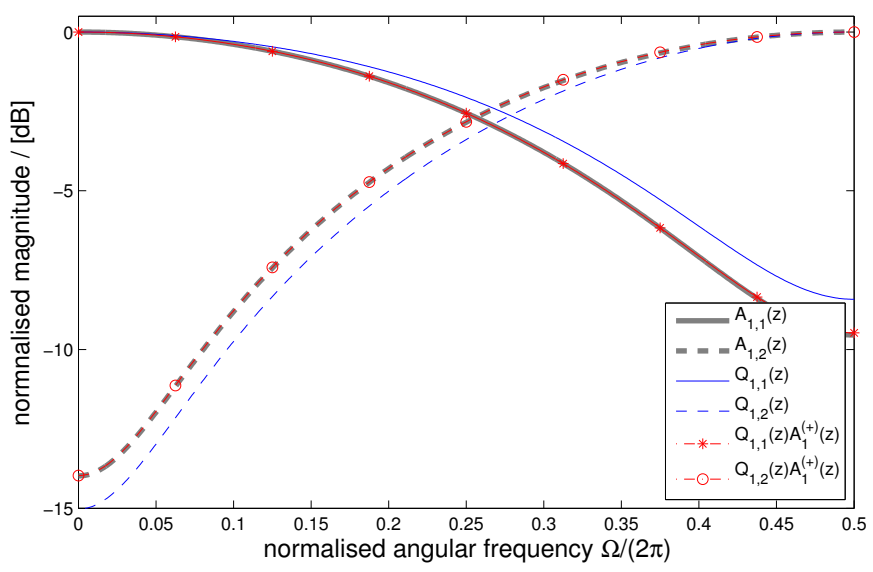

Fig. 3. Transfer functions and principal eigenvector components for measurement campaign $i=1$, with corrected eigenvectors based on both campaigns.

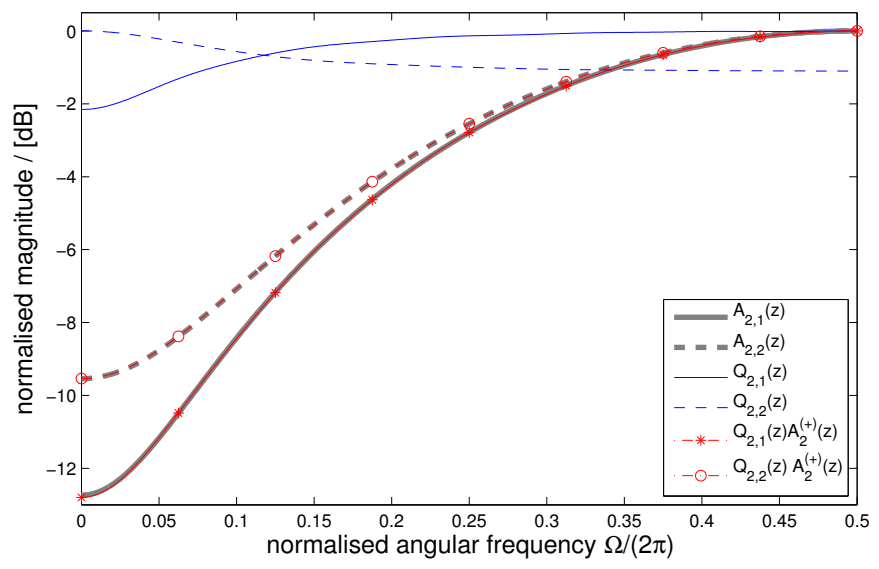

Fig. 4. Transfer functions and principal eigenvector components for measurement campaign $i=2$, with corrected eigenvectors based on both campaigns.

approach, which retains an ambiguity with respect to phase and magnitude even for multiple measurement campaigns due to the negligence of coherence across frequency bins.

Extraction of accurate magnitude responses of the sourcesensor paths depends on robustly determining the greatest common divisor across several polynomials. Estimation errors in the covariance matrices and approximations inherent in iterative PEVD algorithms make this difficult, and robust root finding methods will be crucial for developing this approach further. This may well pose practical limitations for the proposed approach, but does not limit the theoretical findings of this paper.

\section{ACKNOWLEDGEMENT}

This work was supported in parts by the Engineering and Physical Sciences Research Council (EPSRC) Grant number EP/K014307/1 and the MOD University Defence Research Collaboration in Signal Processing.

\section{REFERENCES}

[1] A. Paulraj, R. Roy, and Kailath. A subspace rotation approach to signal parameter estimation. Proc. IEEE, 32(7):984-995, July 1986.

[2] R. O. Schmidt. Multiple emitter location and signal parameter estimation. IEEE Trans. Ant. Prop., 34(3):276-280, Mar. 1986.

[3] H. Wang and M. Kaveh. Coherent signal-subspace processing for the detection and estimation of angles of arrival of multiple wide-band sources. IEEE Trans. ASSP, 33(4):823-831, Aug. 1985.

[4] H. Wang and M. Kaveh. On the performance of signal-subspace processing-part ii: Coherent wide-band systems. IEEE Trans. ASSP, 35(11):1583-1591, Nov. 1987.

[5] M. Alrmah, S. Weiss, and S. Lambotharan. An extension of the MUSIC algorithm to broadband scenarios using polynomial eigenvalue decomposition. In EUSIPCO, pp. 629-633, Barcelona, Spain, Aug. 2011.

[6] V. Reddy, A. Paulraj, and T. Kailath. Performance analysis of the optimum beamformer in the presence of correlated sources and its behavior under spatial smoothing. IEEE Trans. on Acoustics, Speech, and Signal Processing, 35(7):927-936, Jul 1987.

[7] W. Liu. A novel approach to adaptive beamforming for multi-path broadband signals. In IEEE Workshop on Statistical Signal Processing, pages 197-200, Aug 2009.

[8] I. Dokmanić, Y. Lu, and M. Vetterli. Can one hear the shape of a room: the 2-d polygonal case. In IEEE Int. Conference on Acoustics, Speech, and Signal Processing, 2011.

[9] F. Antonacci, J. Filos, M. R. P. Thomas, E. A. P. Habets, A. Sarti, P. A. Naylor, and S. Tubaro. Inference of room geometry from acoustic impulse responses. IEEE Trans. Audio, Speech, and Language Proc., 20(10):2683-2695, Dec. 2012.

[10] L. Remaggi, P. J. B. Jackson, and P. Coleman. Source, sensor and reflector position estimation from acoustical room impulse responses. In 22nd Int. Congress Sound \& Vibration, Florence, Italy, July 2015.

[11] J. G. McWhirter, P. D. Baxter, T. Cooper, S. Redif, and J. Foster. An EVD Algorithm for Para-Hermitian Polynomial Matrices. IEEE Trans. Signal Processing, 55(5):2158-2169, May 2007.

[12] S. Icart and P. Comon. Some properties of Laurent polynomial matrices. In IMA Conf. Maths in Signal Proc., Birmingham, UK, Dec. 2012.

[13] F. Rellich. Störungstheorie der Spektralzerlegung. iii. Mitteilung. Analytische, nicht notwendig beschränkte Störung. Mathematische Annalen, 116:555-570, 1939.

[14] J. Corr, K. Thompson, S. Weiss, I. Proudler, and J. McWhirter. Row-shift corrected truncation of paraunitary matrices for PEVD algorithms. In 23rd Europ. Signal Proc. Conf., pp. 849-853, Nice, France, Aug. 2015.

[15] S. Redif, S. Weiss, and J. McWhirter. Sequential matrix diagonalization algorithms for polynomial EVD of parahermitian matrices. IEEE Trans. Signal Processing, 63(1):81-89, Jan. 2015.

[16] J. G. McWhirter and Z. Wang. Insight into the SBR2 algorithm. In IMA Conf. Maths in Signal Proc., Birmingham, UK, Dec. 2016.

[17] M. Tohidian, H. Amindavar, and A. M. Reza. A dft-based approximate eigenvalue and singular value decomposition of polynomial matrices. EURASIP J Advances in Signal Proc., 2013(1):1-16, 2013.

[18] Euclid. Euclide's elements; the whole fifteen books compendiously demonstrated. [...]. Isaac Barrow, London, 1751.

[19] S. Barnett. Greatest common divisor of several polynomials Math. Proc. Cambridge Philosophical Society, 70:263-268, 91971.

[20] A. Conflitti. On computation of the greatest common divisor of several polynomials over a finite field. Finite Fields and Their Applications, 9(4):423-431, 2003.

[21] D. Henrion and M. Sebek. Reliable numerical methods for polynomial matrix triangularization. IEEE Trans. Aut. Contr., 44(3):497-508, Mar. 1999.

[22] E. Kaltofen, Z. Yang, and L. Zhi. Approximate greatest common divisors of several polynomials with linearly constrained coefficients and singular polynomials. In Proc. International Symposium on Symbolic and Algebraic Computation, pages 169-176, New York, NY, 2006.

[23] P. van Dooren. Rational and polynomial matrix factorizations via recursive pole-zero cancellation. Linear Algebra and its Applications, 137-138(0):663-697, 1990.

[24] A.-G. Wu, G.-R. Duan, and Y. Xue. Kronecker maps and sylvesterpolynomial matrix equations. IEEE Trans. Automatic Control, 52(5):905-910, 2007.

[25] M. Wiesinger-Widi. Sylvester Matrix and GCD for Several Univariate Polynomials. RISC Report Series 11-07, Research Institute for Symbolic Computation, Johannes Kepler University Linz, Austria, May 2011. 